Revue d'histoire de l'enfance « irrégulière »

Le Temps de l'histoire

Hors-série | 2007

Pages d'histoire, la protection judiciaire des mineurs, $\mathrm{XIX} \mathrm{X}^{\mathrm{e}} \mathrm{XX} \mathrm{X}^{\mathrm{e}}$ siècles

\title{
Une maison de correction
}

La colonie de Belle-île-en-Mer 1880-1945

Jacques Bourquin

\section{(2) OpenEdition}

\section{Journals}

Édition électronique

URL : http://journals.openedition.org/rhei/3021

DOI : 10.4000/rhei.3021

ISBN : 978-2-7535-1647-2

ISSN : 1777-540X

Éditeur

Presses universitaires de Rennes

Édition imprimée

Date de publication : 1 juin 2007

Pagination : 259-265

ISSN : 1287-2431

\section{Référence électronique}

Jacques Bourquin, «Une maison de correction », Revue d'histoire de l'enfance « irrégulière » [En ligne]

Hors-série | 2007, mis en ligne le 01 février 2010, consulté le 19 avril 2019. URL : http://

journals.openedition.org/rhei/3021 ; DOI : 10.4000/rhei.3021 


\section{Une maison de correction La colonie de Belle-Île-en-Mer $1880-1945^{(*)}$}

Il y a un questionnement qui traverse en permanence, hier comme aujourd'hui, toute l'histoire de l'intervention auprès de l'enfant qui a à faire avec la justice pénale. Est-il plus un enfant coupable à enfermer, à corriger, qu'un enfant victime à éduquer, à protéger ?

Les réponses varient suivant les époques, et l'histoire de la colonie pénitentiaire de Belle-Île-en-Mer à partir de 1880 et celle de l'IPES après 1945 peuvent apparaître comme les deux phases de cette réponse.

Si à la colonie on parle d'éducation correctionnelle, on y aura surtout des pratiques répressives, disciplinaires. Si l'IPES, soixante-cinq ans plus tard, s’inscrit délibérément dans une voie éducative, il lui faudra de longues années pour se dégager des archaïsmes pénitentiaires.

L'histoire de l'IPES commence à s'écrire, mais celle de la colonie, malgré les travaux très intéressants de M. Garans, nécessite un travail d'archives qui, jusqu’à ce jour, a été à peine entamé.

Avant d'évoquer cette institution, permettez-moi de la situer dans le contexte juridique, social et culturel du XIXème siècle.

La Révolution française, dans une loi de 1791, fixe la minorité pénale à 16 ans. Elle distingue les mineurs discernants, qui seront condamnés à des peines inférieures à celles des adultes, et les mineurs non discernants, qui seront acquittés, éventuellement remis à leurs parents, mais le plus souvent placés dans des maisons d'éducation spéciale pour y être " détenus et élevés ". Il faudra attendre près d'un demi-siècle pour voir ces maisons d'éducation spéciale. En attendant, mineurs discernants et non discernants, quand ils ne sont pas remis à leurs parents, croupiront avec les adultes dans des maisons de détention.

La première réalisation, la maison d'éducation correctionnelle de la Petite Roquette, ouvre à Paris en 1836. Malgré un réel souci d'apprendre un métier
(*) Article publié dans Belle-Îsle histoire, $\mathrm{n}^{\circ} 18$, décembre 1996. Texte de la conférence sur l'histoire de la colonie de Belle-Île donnée par Jacques Bourquin à la mairie du Palais en 1996.

Une maison de correction... / p. 259 à 265 
(1) Expression utilisée fréquemment par Frédéric-Auguste Demetz dans sa correspondance pendant les premières années de la colonie.

(2) Assemblée générale des fondateurs, 1841.

(3) Assemblée générale des fondateurs, 1842.

(4) Loi du 5 août 1850 sur l'éducation et le patronage des jeunes détenus. aux jeunes détenus, cette institution construite sur le modèle du panoptique de Bentham tient beaucoup plus de la prison que de la maison d'éducation.

La seconde réalisation : les colonies agricoles, illustrées par une formule célèbre de l'époque "sauver le colon par la terre et la terre par le colon ». La plus célèbre d'entre elles est ouverte en 1839 à Mettray près de Tours par le magistrat philanthrope Frédéric-Auguste Demetz. Il s'agit pour le fondateur de Mettray d'enlever ceux qu'il appelle " les innocents coupables ", ${ }^{(1)}$ le plus souvent des enfants vagabonds (le vagabondage des mineurs sera un délit jusqu’en 1935), " à la corruption de villes pour les ramener aux principes fondamentaux des sociétés primitives " ${ }^{(2)}$ : la société rurale.

L'essor industriel, l'urbanisation massive, la constitution d'un prolétariat urbain qui inquiète la société bourgeoise conduisent naturellement à ramener les enfants pauvres et vagabonds des villes vers le monde rural, qui acquiert une véritable fonction rédemptrice. Demetz évoquant la nature écrit : "Elle rapproche de Dieu, elle inspire la crainte divine et par là même la soumission à l'autorité. " ${ }^{(3)}$ Un véritable programme d'éducation.

Une loi de 1850 affirme la nécessité de multiplier les colonies agricoles développées par la philanthropie sociale, mais elle dit en même temps : "L'État se substituera à l'initiative privée si le besoin s'en fait sentir " ${ }^{(4)}$; c'est ce qui va se passer dès le milieu du Second Empire.

À partir de 1860, à l'image de l'enfant pauvre et vagabond des villes, se substitue progressivement celle beaucoup plus inquiétante de l'enfant criminel, de l'inamendable. C'est le regard sur l'enfant de justice qui change. On assiste en particulier à un glissement idéologique important qui correspond à l'apparition des théories positivistes qui accordent la priorité aux sciences fondées sur l'observation et rejettent comme utopie l'image de l'enfant de la nature puisée chez Jean-Jacques Rousseau et chez les romantiques. Aux croyances se substituent les certitudes de la science. Le courant positiviste va s'affirmer particulièrement en criminologie où César Lombroso développe l'idée du " criminel né », selon laquelle le délinquant porte sa criminalité dans son propre patrimoine génétique. On va accorder une grande importance à la dégénérescence, aux tares héréditaires. On considère en 1875 le vagabondage comme une forme atavique du fait criminel. Le mineur délinquant est enfermé dans un déterminisme impitoyable. 
L'heure n'est plus aux projets d'éducation ; il n'y a plus pour ce mineur délinquant que le châtiment et l'enfermement. On écrit dans un congrès pénitentiaire de 1895 : «Il faut soumettre l'enfant. S'il continue à fauter, c'est que la discipline n'est pas suffisante. " ${ }^{(5)}$

Comme le prévoyait la loi de 1850, l'Administration pénitentiaire va ouvrir ses propres colonies correctionnelles et pénitentiaires, et entériner le déclin du secteur privé. C'est dans ce contexte que s'ouvre en 1880 la colonie maritime de Belle-Île-en-Mer.

\section{La colonie maritime puis agricole de Belle-Île-en-Mer}

La colonie est créée par décision ministérielle du 29 mai 1880 dans des bâtiments annexes à la citadelle construits en 1848 par le Génie militaire pour y recevoir des détenus politiques. Entre 1872 et 1879 , ce site (HauteBoulogne) recevra des condamnés de la commune.

Pourquoi choisit-on Belle-Île ? Des locaux vacants ? Une insularité qui devrait limiter les évasions ? La réponse est vraisemblablement dans les archives de l'institution déposées aux archives départementales à Vannes et qui, jusqu’à ce jour, ont été très peu travaillées. La colonie pénitentiaire de Belle-Île (c’est son titre officiel) reçoit, selon la loi de 1850, des jeunes détenus acquittés ayant agi sans discernement mais non remis à leurs parents, et des jeunes condamnés à un emprisonnement de six mois à deux ans qui seront pendant les trois premiers mois enfermés dans un quartier distinct. On peut arriver à la colonie à 13 ans et y rester jusqu'à sa majorité pénale, 16 ans, ou sa majorité civile, 21 ans.

C'est " un ensemble austère de longs bâtiments bas sur le bord de la falaise " (6) que décrit l'avocat Henri Rollet qui visitera la colonie en 1892. Ces bâtiments contiennent les bureaux de l'administration, de vastes dortoirs auxquels on accède par des échelles. Ces dortoirs seront, dès la fin du siècle, compartimentés en cellules grillagées de 1,5 mètre sur 2 mètres. Il y a des réfectoires, une buanderie, des ateliers, une chapelle et un quartier disciplinaire de vingt cellules.

Il semble que très rapidement la vocation maritime de la colonie soit très marquée. La section maritime semble regrouper vers 1890 une centaine de colons ; elle est dirigée par un ancien capitaine au long cours auquel sont

(5) Phrase de Maurice

Herbette, directeur de

l'Administration pénitentiaire, citée par J.

Grosmolard in « La lutte contre la criminalité juvénile au XIXème siècle ", publié dans les Archives d'anthropologie criminelle, 1907, vol. 22, p. 94.

(6) Guy Tomel, Henri Rollet, Les enfants en prison, Paris, Plon, 1892, p. 41-42. 
adjoints un ancien capitaine de cabotage et quatre matelots brevetés. Il existe quatre ateliers spéciaux :

- matelotage, timonerie ;

- voilerie, filets ;

- garniture ;

- corderie.

Ce dernier atelier fabrique des cordes pour toute l'Administration pénitentiaire. En 1900, s'ajoute un atelier de sardinerie, ces deux ateliers aidant à la rentabilité de l'institution. Il ne faut pas que ces établissements coûtent cher à la collectivité publique.

Jusqu'en 1895, les colons apprentis matelots font les exercices de marine sur un petit trois-mâts ensablé dans la cour, le Ville de Palais, qui servit à cet effet jusque dans les années 1920.

C'est en 1895 que l'Administration pénitentiaire dote la colonie d'un navire de vingt-cinq mètres, le Sirena, qui peut embarquer vingt colons. Il semblerait qu'entre le 15 juin et le 30 septembre, le Sirena et quelques colons pêchent le thon au large des côtes espagnoles. À cela s'ajoutent plusieurs canots à rames et voiles (le Bangor, le Sauzon, le Locmaria...). Cette vocation maritime des colons fut rapidement remise en cause par l'avocat Rouet qui constate, peut-être à tort, "que les colons sont surtout des jeunes vagabonds des grandes villes peu attirés par la Marine ».

Dès 1887, peut-être même avant, l'Administration pénitentiaire loue, au centre de l'île, une partie du domaine de la famille Trochu pour y installer une section agricole. Ce domaine de Bruté-Souverain, d'une contenance de cent dix-sept hectares, sera définitivement acheté par l'Administration pénitentiaire en 1902. On y construira en 1906 et 1910 deux grands bâtiments de pierre pour y accueillir de nouveaux colons et l'effectif de la colonie atteindra trois cent vingt places.

Au même titre que les colonies publiques de l'époque (Saint-Hilaire, SaintMaurice, Eysses, Aniane), la discipline est extrêmement sévère. L'avocat Henri Rollet, qui deviendra le premier juge des enfants en 1914, écrit en 1892 : «Ce n'est pas à proprement parler une école, c'est un pénitencier. " ${ }^{(7)}$

La violence semble y être quotidienne. Le grave incident du 23 août 1908 en est un exemple. Il est relaté ainsi par la presse de l'époque : «Au large, quatre 
jeunes colons se jettent sur le surveillant Burlut qui était à la barre et le frappent à coups d'aviron. Le crâne défoncé, il est pendu à la drisse du mât. » ${ }^{(8)}$ Les quatre colons furent arrêtés et incarcérés à la prison de Lorient.

Plus tard, en 1924, le directeur de l'Administration pénitentiaire accorde au journaliste Louis Roubaud l'autorisation de visiter les établissements pour mineurs. J'espère, lui dit-il, " que vous allez détruire cette légende de bagnes d'enfants qui court depuis plusieurs années ». ${ }^{(9)}$ Louis Roubaud visite Belle-Île pendant l'été 1924 ainsi que d'autres colonies; le reportage qu'il en tire, Les enfants de Caïn, est un véritable réquisitoire contre ces établissements. Il y a de longues pages consacrées à Belle-Île ; il y évoque ce qu'on appelle le «bal » :

"Une piste ovale très étroite dans une salle couverte où l'on ne doit pas s'écarter de la corde sous peine de tomber. Il y a entre six et vingt "danseurs". La ronde commence à 9 heures du matin et ne s'arrête qu'à 5 heures du soir avec une heure d'interruption pour le déjeuner. L'allure normale est de sept à huit kilomètres à l'heure, elle est entretenue par les surveillants, montre et bâton en main. " ${ }^{(10)}$

Louis Roubaud décrit aussi la corvée de sable : "Vous traversez le terrain militaire, vous descendez à la côte. Il y a un escalier de cinquante marches que vous remontez avec un sac de sable et de galets de trente kilos. Cela pendant de longues heures. " ${ }^{(11)}$

Il conclut son ouvrage en écrivant : "Il faut raser les murs de toutes ces institutions, c'est la seule réponse. " ${ }^{(12)}$

La réponse de l'État fut un décret du 31 décembre $1927^{(13)}$ qui substituait aux termes de " colonies pénitentiaires et correctionnelles " ceux de " maison d'éducation surveillée ". Les surveillants devenaient des moniteurs, mais on ne modifiait rien de leur rôle. Seul changement concret, ils abandonnaient le képi pour le remplacer par une casquette. Les colons devenaient des pupilles et troquaient leurs sabots contre des galoches. Les murs subsistaient, le règlement restait immuable ; en fait, rien n'avait changé.

En 1930, l'introduction du système progressif dans ces institutions n'aura guère d'effet positif. On va diviser les maisons d'éducation surveillée en trois secteurs : épreuve, honneur, excellence. Chaque pupille arrivant est envoyé au secteur épreuve, le plus disciplinaire, puis, en fonction de son amendement, il passera dans des secteurs au régime moins dur. Si l'on couche en « cages à 
poules » au secteur épreuve, on bénéficie d'une " chambrette " au secteur excellence. Mais qu'en est-il de l'amendement ? Est-ce une réelle amélioration morale ou une simple adaptation à la vie institutionnelle ? Ce sera le plus souvent cette dernière référence qui sera privilégiée et ce sont les petits caïds qui se retrouveront en groupe honneur ou excellence.

\section{La révolte de Belle-Île-en-Mer}

\section{Les campagnes de presse. Le début des réformes}

Ces institutions de plus en plus décriées vont faire l'objet de vigoureuses campagnes de presse qui vont alerter l'opinion publique entre 1934 et 1937.

Le point de départ de ces campagnes de presse est lié à une révolte qui éclate à la maison d'éducation surveillée de Belle-Île-en-Mer en août 1934.

Depuis plusieurs mois, le climat est médiocre à l'intérieur de l'institution. Un soir d'août 1934 éclate un incident au réfectoire, un incident apparemment bénin. Contrairement au règlement, un colon a mangé son fromage sans avoir bu sa soupe. Il est puni, ses camarades du réfectoire se solidarisent avec lui, c'est le début d'une révolte que les personnels n'arrivent pas à contenir. Les pupilles franchissent les murs de la colonie, se répandent dans toute l'île où se trouvent, en cette période, beaucoup de vacanciers. L'épisode de la révolte est très connu ; Alexis Danan, le journaliste qui est à l'origine des campagnes de presse, mais qui n'était pas présent sur l'île, l'a raconté de nombreuses fois, en particulier dans ses souvenirs, L'épée du scandale (Robert Laffont, 1961).

Alexis Danan était un journaliste, célèbre à l'époque, qui venait de terminer un reportage sur le bagne de Cayenne dont il réclamait la fermeture. La révolte de Belle-Île était un sujet qui arrivait à point.

On fait appel aux gardes mobiles sur le continent, mais la population de Belle-Île et les vacanciers ramènent de nombreux évadés et, conformément au règlement en usage, reçoivent une prime. Ce fut l'illustration d'un célèbre poème de Prévert, "La chasse à l'enfant "(dans Paroles, Gallimard, 1949), qui fut chanté jusqu’à la veille de la guerre dans les cabarets parisiens. Courant 1937, Marcel Carné et Jacques Prévert tentèrent de tourner un film à partir de la révolte ; ce fut un projet sans lendemain.

Cette campagne de presse se poursuivit trois ans, illustrée par la fermeture de Mettray et de graves incidents à Eysses. 
La ténacité d'Alexis Danan est certes un facteur important de la sensibilisation de l'opinion publique et des pouvoirs politiques au problème des « bagnes d'enfants ". À cela, on peut ajouter une hypothèse très vraisemblable : jamais le chiffre de la délinquance des mineurs n'a été, depuis le début du siècle, aussi bas que vers 1934-1935. La cause en est simple : peu d'enfants sont nés entre 1914 et 1918 ; il y a donc peu d'adolescents, la délinquance juvénile n'inquiète guère et les préoccupations de l'époque sont plus liées à la prévention qu’à la répression.

Tout un courant de réformes va se développer dans ce contexte :

- le décret du 31 octobre 1935 qui va dépénaliser le vagabondage des mineurs en le faisant relever dorénavant de l'assistance éducative, donc du domaine civil ;

- la loi du 13 août 1936 qui réforme la maison d'éducation surveillée de Saint-Maurice ;

- la loi du 12 juillet 1937 qui réforme celle de Saint-Hilaire.

Si une première réforme est tentée à Saint-Maurice en janvier 1937, avec la collaboration du Scoutisme et de l'Éducation nationale, elle aboutira rapidement à un échec, car très mal préparée. Il faudra attendre 1938-1939 pour que la réforme de Saint-Maurice s'engage dans une voie positive avec la collaboration de la direction de l'Enseignement technique. La maison d'éducation surveillée sera avant tout une institution de formation professionnelle.

En fait, c'est un nouveau regard qu'on va porter sur l'enfant « coupable ». Les réformateurs de Saint-Maurice écriront en 1938 : "On ne peut dire que les principes de l'éducation des délinquants soient différents de ceux de l'éducation des enfants normaux, ils sont souvent les mêmes et ne varient que dans leur application. " ${ }^{(14)}$ Cette phrase est importante, car elle réintroduit le mineur délinquant dans une certaine normalité. Il faudra attendre 1945 pour qu'une nouvelle législation de l'enfance délinquante soit promulguée : l'ordonnance du 2 février 1945 qui proclame l'éducabilité du mineur délinquant et par voie de conséquence la création, le 1er septembre 1945, d'une direction de l'Éducation surveillée autonome de l'Administration pénitentiaire.

Ce n'est qu'au début de 1946 que commencera la réforme de l'institution publique d'éducation surveillée de Belle-Île-en-Mer. Depuis avril 1945, l'institution recevait des mineurs anciens miliciens ou ayant appartenu à la division Charlemagne.
(14) Rapport de

Vincent Hourcq, directeur de Saint-Maurice, 1939, cité par Philippe ReyHerme, in Quelques aspects du progrès pédagogique dans la rééducation de la jeunesse délinquante, Paris, Vrin, 1945, p. 136. 